\title{
Anatomical Variations of Cerebral MR Venography: Is Gender Matter?
}

\author{
Gourav Goyal, MD', Rambir Singh, MD², Nikhil Bansal, MD³, Vimal Kumar Paliwal, MD4
}

Purpose: Knowledge of variations in the cerebral dural venous sinus anatomy seen on magnetic resonance (MR) venography is essential to avoid over-diagnosis of cerebral venous sinus thrombosis (CVST). Very limited data is available on gender difference of the cerebral dural venous sinus anatomy variations.

Materials and Methods: A retrospective study was conducted to study the normal anatomy of the intracranial venous system and its normal variation, as depicted by 3D MR venography, in normal adults and any gender-related differences.

Results: A total of 1654 patients (582 men, 1072 women, age range 19 to 86 years, mean age: $37.98 \pm$ 13.83 years) were included in the study. Most common indication for MR venography was headache $(75.4 \%)$. Hypoplastic left transverse sinus was the most common anatomical variation in $352(21.3 \%)$ patients. Left transverse sinus was hypoplastic in more commonly in male in comparison to female $(24.9 \%$ versus $19.3 \%, p=0.009)$. Most common variation of superior sagittal sinus (SSS) was atresia of anterior one third SSS (15, 0.9\%). Except hypoplastic left transverse sinus, rest of anatomical variations of the transverse and other sinuses were not significantly differ among both genders.

Conclusion: Hypoplastic left transverse sinus is the most common anatomical variation and more common in male compared to female in the present study. Other anatomical variations of dural venous sinuses are not significantly differ among both genders.

Key Words : Cerebral venous anatomy; Cerebral venous sinus thrombosis;

Magnetic resonance (MR) venography; Superior sagittal sinus; Straight sinus;

Transverse sinus

\footnotetext{
'Department of Neurology, Mahatma Gandhi Medical College \& Hospital, Jaipur, Rajasthan, India

${ }^{2} \mathrm{MRI}$ Centre, GBH American Hospital, Udaipur, Rajasthan, India

${ }^{3}$ Department of Radiodiagnosis and Imaging, Mahatma Gandhi Medical College \& Hospital, Jaipur, Rajasthan, India

${ }^{4}$ Department of Neurology, SGPGIMS, Lucknow, Uttar Pradesh, India

Received July 19, 2016; accepted after revision August 11, 2016.

Correspondence to: Dr. Gourav Goyal, MD, DM, FINS, Department of Neurology, Mahatma Gandhi Medical College \& Hospital, Jaipur, Rajasthan, India.

Tel. $+91.0141 .2770798 / 2771777 / 2771001-3$

Fax. $+91.0141 .2770303 / 2770900$

E-mail: drgauravjaipur@yahoo.com,drgauravjaipur@gmail.com

This is an Open Access article distributed under the terms of the Creative Commons Attribution Non-Commercial License (http://creativecommons.org/licenses/by-nc/3.0) which permits unrestricted non-commercial use, distribution, and reproduction in any medium, provided the original work is properly cited.
} 
Magnetic resonance venography (MRV) plays important role in studying anatomy of the intracranial venous system and its variation to look for any pathology and misinterpretation of its normal variation $[1,2]$. Visualization of intracranial venous system is important in certain clinical situations such as diagnosis of cortical venous thrombosis and assessment of the patency of the venous sinus lumina encased by meningioma.

It can also be done from various other methods like CT Venogram \& Conventional catheter venography but MR Venography is having overdue benefits like it is non-invasive, does not require ionic radiation exposure or use of any paramagnetic contrast agent administration because of Time of flight (TOF) and Phase Contrast (PC) MR technique, which is most commonly use and provides higher signal to noise and short image time $[3,4]$.

MRV can even evaluate pathology in the vessels which are close to the bony surface which are hard to visualize on CT Angiography due to the artifacts [5]. There are also few disadvantages like it required longer acquisition time but it can be overcome by newly developed parallel imaging technique, due to narrow bore size and loud noise some patients experience claustrophobia which can be overcome by ear plugs and open or wide bore machines, due to the movement it causes artifacts \& signal loss. A number of studies are available on variation of magnetic resonance venography but no literature is available on gender difference of MRV variations in best of our knowledge [3, 6-11].

The purpose of our study is to retrospectively study the normal anatomy of the intracranial venous system and its normal variation, as depicted by $3 \mathrm{D} \mathrm{MRV}$, in normal adults and any gender-related differences.

\section{MATERIALS AND METHODS}

Patients, those were sent for MRV examination in tertiary care teaching hospital from January 2011 to December 2013 were enrolled in this study. MRV data of 2000 patients during study period was retrospectively reviewed. Patients with more than 18 years of age were included in this study. Patients with any congenital or acquired intracranial abnormality, venous thrombosis or previous surgery were excluded from the study. As this study is the retrospective, institutional review board clearance was not sought.

210 patients were excluded because of structural pathology (congenital or acquired) and presence of cortical venous thrombosis. One hundred thirty six patients were 18 or less than 18 years old, therefore excluded. MRV data of 1654 patients were included in final analysis. Clinical indication for MRI and MRV was noted in each patient.

\section{MR Imaging}

MR imaging was performed with superconducting 1.5-T MR machine (Achiva version 1.3; Philips, best the Netherland) and standard head coil. Threedimensional MR venography was performed in the coronal plane by using the following parameters: TE50, TR-500 FOV-230-250, Slice thickness $1 \mathrm{~mm}$, Matrix- $240 \times 256$, flip angle 50 . Additional routine T2WI coronal and axial FLAIR sequences were also performed.

The slice thickness of $1.2 \mathrm{~mm}$ was acquired with contiguous sections using matrix size of $256 \times 256$ and a nominal field of view (FOV) of $27 \mathrm{~cm}$ with TR (Time to Repeat) -22 milliseconds, TE (Time to Echo) 7.4 milliseconds and flip angle of 15o. TRITE- 50/8.4. The velocity encoding applied was $30-40 \mathrm{~cm}$ per second. The images were displayed as 20 maximum intensity projection (MIP) images reconstructed from the source images at 9 degree increments.

\section{Image analysis}

Maximum intensity projections (MIPs) were created at the MR operating console for 3D-MR venography data set. The MIP images were viewed in the sagittal, transverse and coronal planes.

Source images from 3D MRV and MIP images were evaluated for anatomical variations of dural venous sinuses and presence of accessary sinuses. The dural venous sinuses included in this study are superior sagittal sinus, sigmoid sinus, transverse sinus, straight sinus and occipital sinus. The normal variations of transverse and sigmoid sinuses were noted (symmetry, hypoplasia and aplasia or atresia). The transverse sinuses were measured $1 \mathrm{~cm}$ from the Torcula heterophili and the sigmoid sinuses were measured 1 $\mathrm{cm}$ from the transverse sigmoid junctions. Their liner measurement were compared with the superior sagittal sinus. If the linear measurement was less than half the size of the superior sagittal sinus, it was considered hypoplastic and if not visualised it was considered aplastic or atrectic sinus. The internal jugular veins were not included in the study. The presence of arachnoid granulations were recorded. AGs are defined as well-defined CSF-like signal intensity protuberances extending into the dural sinus commonly 


\section{Gourav Goyal, et al.}

associated with adjacent entering cortical veins.

\section{Statistical Analysis}

Numeric values were shown as the mean \pm standard deviation (SD). Chi-square contingency analysis was used to explore the statistically significant difference of MRV variations among both males and females. A difference was considered significant at a $P$ value of less than 0.05 .

\section{RESULTS}

Our study included a total of 1654 patients (582 men, 1072 women, age range 19 to 86 years, mean age: $37.98 \pm 13.83$ years). Mean age for men was $39.44 \pm$

Table 1. Indications for Magnetic Resonance Venography (MRV)

\begin{tabular}{lcc}
\hline Indication & Male (\%) & Female (\%) \\
\hline Headache & $453(77.8)$ & $794(74.1)$ \\
Headache and vertigo & $67(11.5)$ & $150(14.0)$ \\
Seizure & $23(4.0)$ & $64(6.0)$ \\
Headache and vomiting & $19(3.3)$ & $34(3.2)$ \\
Vertigo & $12(2.1)$ & $21(2.0)$ \\
Altered sensorium & $1(0.2)$ & $6(0.6)$ \\
Forgetfulness & $4(0.7)$ & $1(0.1)$ \\
\hline Others & $3(0.5)$ & $2(0.2)$ \\
\hline
\end{tabular}

14.46 (range 19 to 86 years) and for women $37.19 \pm$ 13.41 (range 19 to 85 years).

\section{Indications}

Most common indication for MRV was headache (75.4\%). Other indications were headache and vertigo (13.1\%), Seizure (5.3\%), Headache and vomiting (3.2\%), vertigo $(2 \%)$, altered sensorium $(0.5 \%)$, forgetfulness $(0.3 \%)$ and others $(0.2 \%)$. Indications for MRV in both sexes have been summarized separately in Table 1 .

\section{Transverse and sigmoid sinus}

Of the 1654 MR venograms obtained, the transverse sinus was found to be symmetrical in 1106 (66.9\%) patients. Left transverse sinus was hypoplastic in 352 $(21.3 \%)$ and aplastic/atrectic in 67 (4.1\%) cases. Right transverse sinus was hypoplastic in $91(5.5 \%)$ and aplastic/atrectic in $12(0.7 \%)$ patients. $1.6 \%$ cases had bilateral hypoplastic transverse sinuses. In comparison, female had significantly more symmetrical transverse sinus than male $(69.2 \%$ versus $62.5 \%$, p value, 0.007$)$. Left transverse sinus was hypoplastic in more commonly in male in comparison to female $(24.9 \%$ versus $19.3 \%$ ). This difference is statistically significant ( $p$ value, 0.009). Rest of normal variations of transverse sinus are not different in both the genders significantly.

$1418(85.7 \%)$ cases had symmetrical sigmoid sinuses. Left sigmoid sinus was hypoplastic or

Table 2. Normal Variations of Transverse and Sigmoid Sinuses

\begin{tabular}{|c|c|c|c|c|}
\hline Variation & Total (\%) & Male (\%) & Female (\%) & $\mathrm{p}$ value \\
\hline \multicolumn{5}{|l|}{ Transverse sinus } \\
\hline Symmetrical & $1106(66.9)$ & $364(62.5)$ & $742(69.2)$ & 0.007 \\
\hline Left sided hypoplastic & $352(21.3)$ & $145(24.9)$ & 207 (19.3) & 0.009 \\
\hline Left sided aplastic/Atrectic & $67(4.1)$ & $31(5.3)$ & $36(3.4)$ & 0.07 \\
\hline Right sided Hypoplastic & $91(5.5)$ & $33(5.7)$ & $58(5.4)$ & 0.91 \\
\hline Right sided aplastic/Atrectic & $12(0.7)$ & $4(0.7)$ & $8(0.7)$ & 0.87 \\
\hline Hypoplastic Bilateral Transverse sinuses & $26(1.5)$ & $5(0.9)$ & $21(2.0)$ & 0.13 \\
\hline \multicolumn{5}{|l|}{ Sigmoid sinus } \\
\hline Symmetrical & $1418(85.7)$ & $483(83.0)$ & 935 (87.2) & 0.02 \\
\hline Left sided Hypoplastic & $154(9.4)$ & $66(11.3)$ & $88(8.2)$ & 0.05 \\
\hline Left sided aplastic/atrectic & $35(2.1)$ & $15(2.6)$ & $20(1.9)$ & 0.43 \\
\hline Right sided Hypoplastic & $40(2.4)$ & $17(2.9)$ & $23(2.1)$ & 0.42 \\
\hline Right sided Aplastic/Atrectic & $4(0.2)$ & $1(0.2)$ & $3(0.3)$ & 0.92 \\
\hline Hypoplastic Bilateral Sigmoid sinuses & $3(0.2)$ & $0(0)$ & $3(0.3)$ & 0.50 \\
\hline
\end{tabular}


aplastic/atrectic in 11.4\% (189) cases. Right sigmoid sinus was hypoplastic in 40 (2.4\%) and aplastic/atrectic in $4(0.2 \%)$. Three patients had hypoplastic bilateral sigmoid sinuses. Similar to transverse sinus, sigmoid sinus was symmetrical more commonly in female than male $(87.2 \%$ versus $83 \%, p=0.02)$. Difference between male and female for other variations of sigmoid sinus is not statistically significant. Anatomical variations and gender difference transverse and sigmoid sinuses have been summarized in Table 2 .

\section{Superior sagittal sinus}

$1616(97.7 \%)$ patients had normal superior sagittal sinus. Most common variation of SSS was atresia of anterior one third SSS $(15,0.9 \%)$. Other variations were hypoplasia of middle part of SSS $(13,0.7 \%)$, hypoplasia of anterior one third of SSS $(6,0.4 \%)$, hypoplasia of anterior $2 / 3 \mathrm{rd}$ of SSS $(3,0.2 \%)$ and hypoplasia of anterior half of SSS $(1,0.1 \%)$. There was no statistically significant difference among males and females for these variations.

Flow gap in SSS was noted in 5 patients. Three cases had flow gap in posterior $1 / 3 \mathrm{rd}$ of SSS and $2 \mathrm{had}$ in middle part of SSS. Anatomical variations and gender difference of superior sagittal sinus have been summarized in Table 3.

\section{Accessory findings}

Straight sinus (SS) was hypoplastic in $4(0.2 \%)$ patients $(1(0.2 \%)$ male and $3(0.3 \%)$ female $)$. Difference among both genders is not statistically significant $(p=0.92)$. No duplication or triplication of $\mathrm{SS}$ is noted in study population. The occipital sinus was identified in $23(1.4 \%)$ patients. Oblique occipital sinus was noted in $13(0.8 \%)$ patients. Five $(0.9 \%)$ men and $18(1.7 \%)$ women had occipital sinus. Gender difference for occipital sinus is not statistically significant ( $p$ value $=0.25$ ). Arachnoid granulations were noted in 47 $(2.8 \%)$ patients. Most common site for AGs was the lateral aspect of left TS $(22,1.3 \%)$. Other sites were the lateral aspect of right TS $(10,0.6 \%)$, middle part of SSS $(9,0.5 \%)$, posterior $1 / 3$ of SSS $(3,0.2 \%)$, lateral aspect of bilateral TS $(2,0.1 \%)$ and left sigmoid sinus $(1,0.7 \%)$. Normal anatomical variations of cerebral dural venous sinuses on MRV have been illustrated in Figure 1.

\section{DISCUSSION}

The study was conducted to evaluate the normal anatomical variations in intracranial venous sinuses and gender differences of normal variations in the western Indian population. Most common indication to get MRV of brain was headache in this study. Hypoplastic left transverse sinus was the most common anatomical variation, predominantly in male compared to female. Other anatomical variations of the transverse and sigmoid sinuses were not significantly differ among both genders. Atresia of anterior one third of SSS was the most common variation of SSS, though not different among males and females.

Transverse sinus abnormalities were described by Alper et al [6]. Symmetrical transverse sinuses were reported in 31\%. Left transverse sinus was hypoplastic in 39\% and aplastic in $20 \%$ of cases. Right transverse sinus was hypoplastic in $6 \%$ and aplastic in $4 \%$. In other study of 100 patients, $10 \%$ had symmetrical transverse sinuses, 35\% hypoplastic left transverse sinus, $13 \%$ hypoplastic right transverse sinus and $1 \%$ had aplastic left transverse sinus [10]. In contrast, our study showed symmetrical transverse sinus in $66.9 \%$, hypoplastic left transverse sinus in $21.3 \%$ and hypoplastic right transverse sinus in $5.5 \%$. This difference in our study can be attributed to ethnical difference of the population.

The most frequent SSS variation is the hypoplasia of rostral third of the SSS second only to preferential drainage of SSS to one of the transverse sinus $[12,13]$.

Table 3. Normal Variations of Superior Sagittal Sinus (SSS)

\begin{tabular}{|c|c|c|c|c|}
\hline & Total (\%) & Male & Female & $p$ value \\
\hline Normal & $1616(97.7)$ & $569(97.8)$ & $1047(97.7)$ & 0.96 \\
\hline Anterior one third hypoplastic & $6(0.4)$ & $3(0.5)$ & $3(0.3)$ & 0.74 \\
\hline Atractic anterior one third & $15(0.9)$ & $6(1.0)$ & $9(0.8)$ & 0.90 \\
\hline Hypoplastic Anterior Half of SSS & $1(0.1)$ & $0(0)$ & $1(0.1)$ & 1.00 \\
\hline Hypoplastic anterior $2 / 3^{\text {rd }}$ of SSS & $3(0.2)$ & $1(0.2)$ & $2(0.2)$ & 0.59 \\
\hline Hypoplastic middle part of SSS & $13(0.7)$ & $3(0.5)$ & $10(0.9)$ & 0.53 \\
\hline
\end{tabular}


Kaplan and Browder reported hypoplastic rostral SSS in 7 of $382(1.8 \%)$ anatomic specimens in 1 series and in 12 of $201(6 \%)$ specimens in a second anatomic series [14]. In the presence of complete hypoplastic rostral SSS, a pair of large parasagittal superior frontal cortical veins that run dorsally to join the origin of the SSS close to the coronal suture replace the absent rostral portion of the SSS $[12,13]$. In a series of 100 patients, hypoplasia of rostral portion of SSS was studied by CT angiography. Complete hypoplastic rostral SSS was reported in 3\% of patients [15]. In other study, this abnormality was reported in $9 \%$ of cases [10]. In our study, atresia of SSS was noted in $0.9 \%$ and hypoplasia in $0.4 \%$. Variation in incidence of hypoplastic or atrectic rostral third of SSS in different studies can be explained by use of different techniques or variation in inclusion criteria. Flow gaps in SSS and partial split SSS were reported in $24 \%$ and $12 \%$ in a study [10]. In other study, short segment flow gaps in SSS were observed in 5\% of cases [11]. In our study, flow gaps were noted in 5 patients.

The occipital sinus, the smallest of the dural venous sinuses, which may be solitary, duplicated, or composed of a mesh of venous collaterals, is contained within the attached margin of the falx cerebelli and connects the torcula with the IJV [16]. Occipital sinuses were reported from 4 to $35.5 \%$ of cases in different studies $[2,3,11,17]$. In a study of 100 children, persistent occipital sinuses were seen in $13 \%$ of patients less than 25 months of age but in only $2 \%$ of children older than 5 years [9]. This study support the hypothesis of involution of occipital sinus once most of
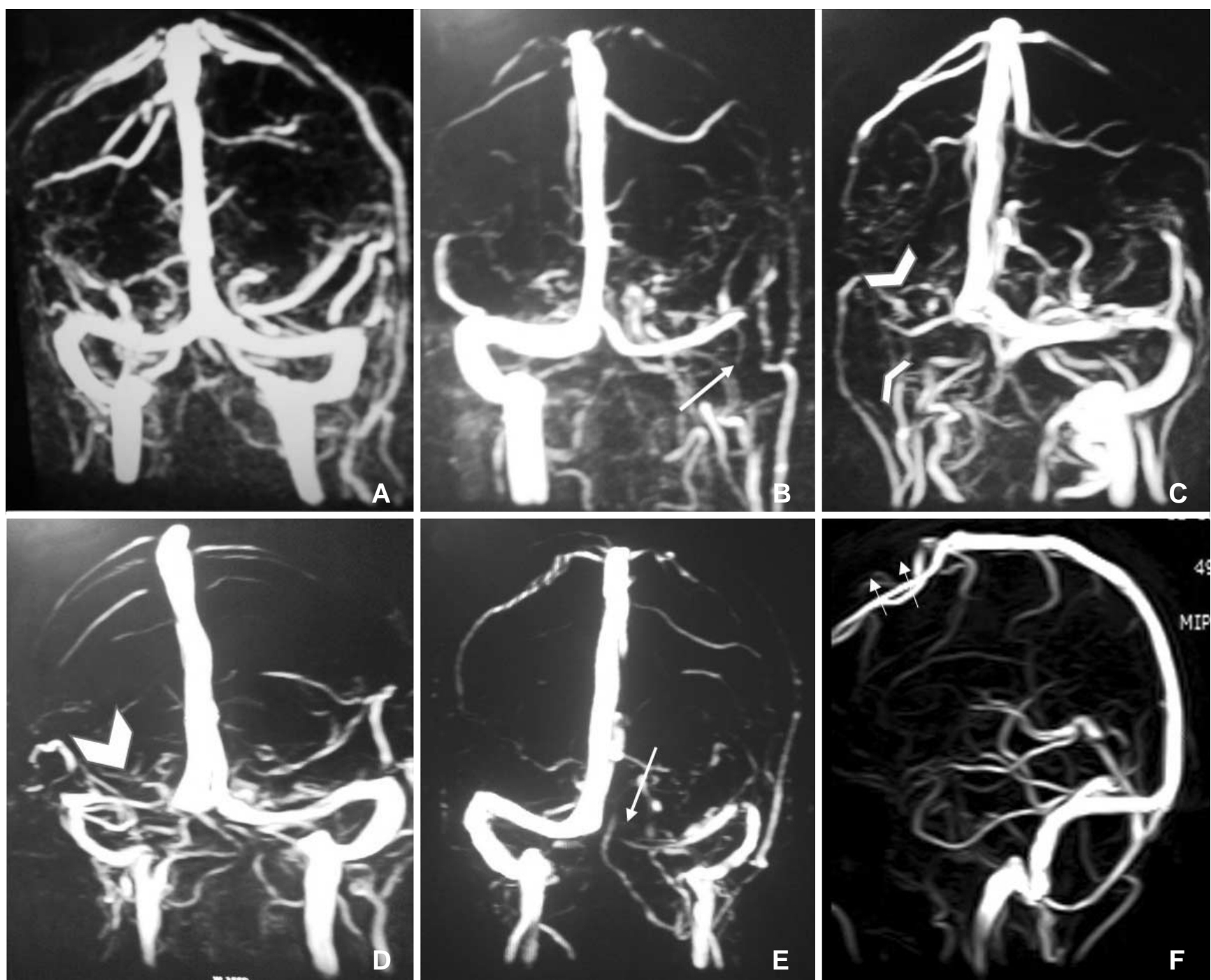

Fig. 1. A. Normal MRV including symmetrical transverse sinuses. B. Hypoplastic left transverse sinus and aplastic left sigmoid sinus (long thin arrow). C. Aplastic right transverse and sigmoid sinus (arrowheads). D. Hypoplastic right transverse sinus (thick arrowhead). E. Aplastic left transverse sinus (long thin arrow) and hypoplastic left sigmoid sinus. F. Aplastic anterior one third superior sagittal sinus (short thin arrows). 
the venous flow passes through the large dural sinuses as the child achieves an upright position. In our study, occipital sinus was identified in $1.4 \%$ of the patients. Over reported incidence of the occipital sinus in the literature may be attributed to wrong interpretation of other venous structures as occipital sinus or ethnical differences of studied populations. Accurate information of the incidence of occipital sinuses can be accumulated by future autopsy series.

\section{Gender difference}

The prevalence and symptomatology of many neurological and psychiatric conditions differ substantially between males and females. In a meta-analysis regarding sex differences in human brain morphology, differences in overall brain volumes are found to be sustained between males and females from newborns to individuals over 80 years old [18]. On average, males have larger intracranial volume (ICV) $(12 \%)$, total brain volume (TBV) $(11 \%)$, cerebrum $(\mathrm{Cb})(10 \%)$, grey matter (GM) (9\%), white matter (WM) (13\%), cerebrospinal fluid (CSF) $(11.5 \%)$ and cerebellum (Cbl) (9\%) absolute volumes than females [18]. Difference between different brain structures among both genders can explain predisposition of some neuropsychiatric disorders to specific gender. Limited data is available on difference of intracranial vasculature among both the genders. In a small study of MR angiography of 30 cases, males were found to have larger diameter of posterior circulation arteries (posterior cerebral artery and basilar artery) compared to females [19]. Though there was no significant difference in anterior circulation arteries among males and females. Anatomic variations of sigmoid sinuses on phase contrast MR angiography were studied in a study of 257 cases of different age groups (5-82 years) [20]. Among 257 cases, 91 were males and 166 were females. In female group, frequency of reduction of flow through right sigmoid sinus is slightly higher than in male group. Frequency of reduction of flow through left sigmoid sinus increase as age increase in male group. In female group frequency of reduction of flow through right sigmoid sinus increase as age increase. In our study, females were more likely to have symmetrical transverse and sigmoid sinuses than males. Males had more hypoplastic left transverse sinus compared to females. Significance of gender difference of intracranial venous sinuses anatomy is not known.

The strength of the present study is large sample size, still retrospective nature of the study is the major limitation. Other limitations are single center study, absence of contrast-enhanced MRV and lack of long term follow up data to look for significance of anatomical variations of MRV. Single center study may cause case selection bias and may miss more variations. Reporting by single radiologist in the present study may also cause bias in the interpretation. Venous flow in the plane of image acquisition may produce saturation and resultant nulling of the venous signal at TOF MR Venography, may lead to misinterpretation of images and diagnosis. Visualization of the dural sinuses is superior with contrast-enhanced MR venography because of a decrease in the effects of turbulent flow. Absence of contrast-enhanced MRV in the present study may overinterpret the flowgaps or other anatomical variations of the dural sinuses. Future prospective studies with contrast-enhanced MRV or autopsy series should be planned to overcome these limitations.

\section{CONCLUSION}

Knowledge of anatomical variations of cerebral dural venous sinuses is important. In the absence of this, flow gap in the venous sinus, hypoplasia and aplasia of the transverse sinus may be mistaken for venous sinus thrombosis. Hypoplastic left transverse sinus is the most common anatomical variation in the present study. Hypoplastic left transverse sinus is more common in male compared to female. Other anatomical variations of dural venous sinuses are not significantly differ among both genders. Present study is the largest study from India and first in the literature to study the gender difference of anatomical variations of dural venous sinuses in best of our knowledge. Importance of male predominance of hypoplastic transverse sinus is not clear. Further studies are needed to explore the importance of gender difference of anatomical variations of dural venous sinuses and to confirm the results of present study.

\section{Acknowledgments}

We are acknowledging Dr. Rekha Gupta for her help.

\section{References}

1. Mattle HP, Wentz KU, Edelman RR, Wallner B, Finn JP, Barnes P, et al. Cerebral venography with MR. Radiology 1991;178:453458

2. Ayanzen RH, Bird CR, Keller PJ, McCully FJ, Theobold MR, Heiserman JE. Cerebral venography: normal anatomy and potential diagnostic pitfalls. AJNR Am J Neuroradiol 2000;21:7478

3. Widjaja E, Griffiths PD. Intracranial MR venography in children: 


\section{Gourav Goyal, et al.}

normal anatomy and variations. AJNR Am J Neuroradiol 2004;25:1557-1562

4. Liauw L, van Buchem MA, Spilt A, de Bruine FT, van den Berg $\mathrm{R}$, Hermans J, et al. MR angiography of the intracranial venous system. Radiology 2000;214:678-682

5. Wetzel SG, Kirsch E, Stock KW, Kolbe M, Kaim A, Radue EW. Cerebral veins: comparative study of CT venography with intraarterial digital subtraction angiography. AJNR Am J Neuroradiol 1999;20:249-255

6. Alper F, Kantarci M, Dane S, Gumustekin K, Onbas O, Durur I. Importance of an anatomical asymmetries of transverse sinuses: an MR venographic study. Cerebrovasc Dis 2004;18:236-239

7. Cure JK, Van Tassel P, Smith MT. Normal and variant anatomy of the dural sinuses. Semin Ultrasound CT MR 1994;15:499-519

8. Zouaoui A, Hidden G. Cerebral venous sinuses: anatomical variants or thrombosis? Acta Anat (Basel) 1988;133:318-324

9. Rollins N, Ison C, Booth T, Chia J. MR venography in the pediatric patient. AJNR Am J Neuroradiol 2005;26:50-55

10. Surendrababu NR, Subathira, Livingstone RS. Variations in the cerebral venous anatomy and pitfalls in the diagnosis of cerebral venous sinus thrombosis: low field MR experience. Indian J Med Sci 2006;60:135-142

11. Sharma UK, Sharma K. Intracranial MR venography using lowfield magnet: normal anatomy and variations in Nepalese population. JNMA J Nepal Med Assoc 2012;52:61-65

12. Kaplan HA, Browder AA, Browder J. Atresia of the rostral superior sagittal sinus: associated cerebral venous patterns. Neuroradiology 1972;4:208-211
13. Hacker H. Superficial supratentorial veins and dural sinuses. In: Newton TH, Gordon Potts MDD, eds. Radiology of the Skull and Brain: Angiography. St. Louis: C.V. Mosby Company; 1974: 1851-902

14. Kaplan HA, Browder J. Atresia of the rostral superior sagittal sinus: substitute parasagittal venous channels. J Neurosurg 1973;38:602-607

15. San Millán Rúiz D, Fasel JH, Gailloud P. Unilateral hypoplasia of the rostral end of the superior sagittal sinus. AJNR Am J Neuroradiol 2012;33:286-291

16. San Millán Ruíz D, Gailloud P, Rufenacht DA, Delaville JH, Henry F, Fasel J. The craniocervical venous system in relation to cerebral venous drainage. AJNR Am J Neuroradiol 2002;23:15001508

17. Lang J. The fl oor of the posterior cranial fossa. In: clinical Anatomy of the posterior cranial fossa and its foramina. New York: Thieme; 199:6-9

18. Ruigrok AN, Salimi-Khorshidi G, Lai MC, Baron-Cohen S, Lombardo MV, Tait RJ, et al. A meta-analysis of sex differences in human brain structure. Neurosci Biobehav 2014;39:34-50

19. Stefani MA, Schneider FL, Marrone ACH, Severino AG. Influence of the gender on cerebral vascular diameters observed during the magnetic resonance angiographic examination of willis circle. Braz Arch Biol Technol? 2013;56:45-52

20. Savelyeva L, Bogomyakova O, Prygova Y, Tulupov A. Anatomic variations of sigmoid sinuses on phase contrast MR-angiography. Paper presented at: ECR 2012. European Congress of Radiology; 2012 March 1 - 5, 2012; Vienna, Austria. 